\title{
Implications of Regional Anaesthesia for Favourable Postoperative Outcomes
}

\author{
Abhijit Nair, ${ }^{1,2}$ Sandeep Diwan ${ }^{3}$ \\ ${ }^{1}$ Department of Anaesthesia, Basavatarakam Indo-American Cancer Hospital \& Research Institute, Hyderabad, Telangana State, India. \\ ${ }^{2}$ Department of Anaesthesiology, Ibra Hospital, North Sharqiya Governorate, Ibra-414, Sultanate of Oman. \\ ${ }^{3}$ Department of Anaesthesia, Sancheti Hospital, Pune, Maharashtra, India.
}

\section{Introduction}

Patient centered outcomes after surgery are described in terms of improving quality of life and functional status, prevent cognitive impairment, delirium, anxiety and depression and preserve organ function [1]. Regional anaesthesia (RA) when used solely or for postoperative analgesia with general anaesthesia (GA) indeed provides better quality of analgesia, lesser opioid consumption and lesser adverse events like postoperative nausea/vomiting (PONV) due to opioids, bleeding or renal toxicity (due to non-steroidal anti-inflammatory drugs). However, the benefits of RA are not just confined to providing opioid-sparing analgesia but many other important early and late postoperative outcomes which has established RA as an integral part of perioperative analgesia [2]. In this editorial, the term RA is used for central neuraxial blocks (spinal and epidural anaesthesia), the peripheral nerve blocks and the fascial plane blocks depending on the type of surgery and the purpose of RA i.e., surgical anaesthesia or postoperative pain relief.

\section{Acute Pain Management}

The list of advantages of providing good quality postoperative analgesia is endless. The few most important advantages are patent comfort, early mobilization, hastened wound healing process, reduced risk of thromboembolic phenomenon like deep vein thrombosis, early recovery of gut function, lesser duration of hospital stay and thus overall lesser cost of treatment [3]. Poorly managed postoperative pain has been found responsible for development of chronic postsurgical pain after a few months [4]. Needless to say, the above listed advantages of acute pain management are absent in poorly managed pain. Delayed mobilization, cardiopulmonary issues, prolonged hospital stay and an overall increased cost of treatment are the sequelae to poorly managed postoperative pain.

The paper by Kessler et al reviewed the existing evidence for the efficacy of peripheral RA by performing a systematic literature search of all prospective studies [5]. Authors analyzed short- and long-term benefits of RA along with complications that were reported. The RA techniques reviewed were upper extremity blocks (interscalene, supraclavicular, infraclavicular, axillary), lower extremity blocks (femoral, saphenous, sciatic, psoas compartment) and truncal blocks (cervical, intercostal, ilioinguinal/ iliohypogastric, transversus abdominis plane block, rectus sheath block). Authors concluded that all blocks led to improved patient comfort and satisfaction, with good postoperative analgesia and negligible nerve damage with the exception of TAP blocks for which authors felt the evidence was insufficient and conflicting. However, authors also suggested further studies to ascertain long term benefits like improved functional outcomes conferred by these RA interventions.

Adding RA to acute pain management especially thoracic epidural analgesia reduces excessive sympathetic activity, reduces myocardial oxygen demand and thus prevents myocardial ischemia in susceptible patients. RA also preserves hormonal milieu by preventing the surge of catabolic hormones like cortisol, adrenocorticotrophic hormone, growth hormone, catecholamines, angiotensin 2, and glucagon all of which are due to surgical stress response. RA improves cough, prevents atelectasis, prevents ventilation: perfusion mismatch, improves gut motility and thromboembolism [6].

Persistent acute pain leads to increased catecholamines, increased activation of renin-angiotensin-aldosterone system, antidiuretic hormone increased excretion of potassium all which leads to sodium and water retention which has detrimental outcomes [7]. The benefits of RA conferred on cardiovascular, respiratory, gastrointestinal and other important systems in the body is depicted in Table 1.

Address of Correspondence: Dr. Abhijit Nair, Basavatarakam Indo-American Cancer Hospital \& Research Institute, Hyderabad, Telangana State, India. Ibra Hospital, North Sharqiya Governorate, Ibra-414, Sultanate of Oman.E-mail: abhijitnair95@gmail.com

DOI: 10.13107/ijra.2021.v02i01.019| (C) 2021 International Journal of Regional Anaesthesia | Available on www.ijrajournal.com | This is an Open Access article distributed under the terms of the Creative Commons Attribution Non-Commercial License (http://creativecommons.org/licenses/by-nc/3.0) which permits unrestricted non-commercial use, distribution, and reproduction in any medium, provided the original work is properly cited.

How to cite this article: Nair A, Diwan S | Implications of Regional Anaesthesia for Favourable Postoperative Outcomes | International Journal of Regional Anaesthesia | January-June 2021; 2(1): 13-16. 


\section{Postoperative Nausea/Vomiting}

Pure RA techniques without opioid adjuncts can reduce PONV. However, with RA solely or as a part of MMA, intraoperative volatile anaesthetics and perioperative consumption could be less and thus can reduce the incidence of PONV especially in susceptible patients. [8] In the fourth consensus guidelines for management of PONV published recently in Anesthesiology (2020), RA techniques like thoracic epidural analgesia and fascial plane blocks has been found to reduce PONV provided opioids are not used as adjuncts [9].

\section{Thromboembolic Phenomenon}

Neuraxial blocks have been known to have antithrombotic effect in the deep veins thereby prevents deep vein thrombosis which if develops postoperatively can have deleterious cardiopulmonary outcome $[10,11]$. It has been shown that there are decreased odds of thromboembolic events with neuraxial blocks when compared to general anaesthesia alone (odds ratio- 0.79; 99\% confidence interval, 0.68-0.91) [12]. In a systematic review by Johnson et al, authors compared general with neuraxial anaesthesia in patients undergoing total hip and knee arthroplasties. Authors found that when compared with general anaesthesia, neuraxial anaesthesia was associated with lower risk of deep vein thrombosis (RR 0.51; 95\% CI 0.41-0.62) based on nine studies and pulmonary embolism (RR 0.36; 95\% CI 0.22-0.600 based on seven studies in patients who did not receive chemical antithrombotic prophylaxis. However, when pharmacological antithrombotic prophylaxis was a part of perioperative plan, there were no statistically significant differences in either deep vein thrombosis or pulmonary embolism rates after hip arthroplasties [13].

\begin{tabular}{|c|c|}
\hline System concerned & Benefits \\
\hline Respiratory & $\begin{array}{l}\text { Facilitates cough and expectoration } \\
\text { Prevents atelectasis, prevents hypoxemia } \\
\text { Preserves functional residual capacity } \\
\text { Early extubation after major abdominal/thoracic surgeries } \\
\text { Prevents ventilation: perfusion mismatch }\end{array}$ \\
\hline Cardiovascular & $\begin{array}{l}\text { Prevents increased myocardial oxygen demand } \\
\text { Reduces sympathetic activity }\end{array}$ \\
\hline Renal & $\begin{array}{l}\text { Reduces sympathetic activity and reduces renin-angiotensin } \\
\text { aldosterone activity reduces fluid/water retention and } \\
\text { thus prevents electrolyte disturbances }\end{array}$ \\
\hline Gastrointestinal & Prevents postoperative ileus, improves peristalsis \\
\hline Central nervous system & $\begin{array}{l}\text { Good analgesia, less opioids/anaesthetics, better sleep, } \\
\text { early discharge and lesser hospital stay }\end{array}$ \\
\hline Endocrinological & $\begin{array}{l}\text { Better glycaemic control } \\
\text { (due to attenuation of hyperglycaemic response to stress) }\end{array}$ \\
\hline Immunological & $\begin{array}{l}\text { Opioid sparing, reduction in volatile anaesthetic use: } \\
\text { preserves immunity, could prevent cancer recurrence- } \\
\text { currently no definitive data }\end{array}$ \\
\hline Others & $\begin{array}{l}\text { Prevents lipolysis } \\
\text { Preserves muscle mass } \\
\text { Reduces postoperative nausea/vomiting }\end{array}$ \\
\hline
\end{tabular}

Table 1: showing benefit of utilizing regional anaesthesia on various systems 


\section{Chronic Pain}

Chronic postsurgical pain (CPSP) is defined as an unpleasant sensory and emotional experience that persists for 3 to 6 months after surgery [14]. In a Cochrane systematic review and metaanalysis by Andreae et al, authors described the importance of thoracic epidural analgesia and thoracic paravertebral block in preventing persistent postoperative pain after thoracic surgery and breast surgery respectively. However, the limitations of this review were small numbers, performance bias, attrition, and incomplete outcome data especially at 12 months. Authors also cautioned that conclusions of this review should not be extrapolated to other surgical interventions or regional anaesthesia techniques. Postamputation limb pain or phantom limb pain (PLP) develops as a result of complex interplay of peripheral and central sensitization. It has been revealed that pain mechanisms involved during the early phase following amputation and with chronic PLP are different. Well-controlled pain preoperatively using an epidural or with intravenous patient-controlled analgesia initiated 48 hours prior to amputation and by continuing this for 48 hours postoperatively has shown decreased PLP at 6 months [15].

\section{Postoperative cognitive dysfunction (POCD)}

Postoperative delirium is a transient and fluctuating disturbance of consciousness that tends to occur shortly after surgery. However, POCD is a more persistent problem of a change in cognitive performance as assessed by neuropsychological tests [16]. POCD is more common and stormy in elderly than young patients [17]. In a systematic review and meta-analysis by Mason et al, authors in the conclusion suggested that use RA wherever possible especially in people otherwise vulnerable to developing cognitive symptoms [18].

Based on current evidence there is no definitive comparative data showing that either general or regional anaesthesia is associated with a reduced risk for the development of cognitive dysfunction following surgery in susceptible patients [19].

In a systematic review by Zywiel et al were authors investigated influence of anaesthesia and pain management on cognitive dysfunction after joint arthroplasty, authors concluded that nonopioid postoperative pain management techniques, limiting narcotics to oral formulations and avoiding morphine, appear to reduce the risk of postoperative cognitive dysfunction. This is only possible when RA is utilized for postoperative pain management even though current evidence is limited [20].

\section{RA and Cancer Recurrence}

There is enough evidence which has demonstrated that use of perioperative opioids and inhalational anaesthetics are detrimental in patients undergoing oncological surgeries. Opioids and volatile anaesthetics affect the function of the protective natural killer (NK) cells, causes immunosuppression which later promotes cancer recurrence. Although the data analysed is from retrospective and animal studies, the concern is imperative. Adding RA for oncosurgeries can have a favorable effect in postoperative outcomes. RA would reduce perioperative opioid and intraoperative volatile anaesthetic consumption and thus theoretically has an edge when compared to pure general anaesthesia technique [21-23]. However, Sessler et al recently presented the results of a multicentric randomized controlled trial (13 hospitals from various countries) which enrolled 2132 patients undergoing breast cancer surgeries. They solemnly concluded that regional anaesthesia-analgesia (paravertebral block and propofol) did not reduce breast cancer recurrence after potentially curative surgery compared with volatile anaesthesia (sevoflurane) and opioids. Moreover, the frequency and severity of persistent incisional breast pain was unaffected by anaesthetic technique [24].

Use of USG in RA has revolutionized perioperative care. Newer blocks are being described and its comparison with a gold standard or an immediate predecessor is keeping RA researchers busy. Although US has helped in reducing volume of LA used for achieving a successful block, the complications associated with RA like postoperative neurological symptoms, local anaesthesia systemic toxicity, hemi diaphragmatic paresis and pneumothorax are still getting reported in registries and literature [25].

Topor et al have described several strategies which can be applied while performing various RA techniques so as to reduce nerve injuries [26]. Having an awake patient, use of USG and neurostimulation, using a short-beveled echogenic needle, avoiding paresthesia, avoid intraneural injections by using in-line pressure monitoring, lowest possible LA and avoiding adrenaline while injecting around nerves with poor vascularity (sciatic nerve).

The second edition of International journal of regional anaesthesia (IJRA) which is the official journal of Academy of Regional Anaesthesia-India (AORA-INDIA) is covering topics like anatomy of brachial plexus above clavicle, current understandings on local anaesthetic volume in blocks above clavicle, cadaveric workshops and its importance in RA practice, an update on abdominal wall blocks for abdominal surgeries, regional anaesthesia techniques for breast surgeries, and landmark-guided and neurostimulation-guided forearm blocks [27-32]. The issue also has articles showcasing the details of AORA mobile application, sterility during RA and also a topic on how to write case reports [33-35].

\section{Conclusion}

Every patient deserves good quality postoperative pain relief based on current recommendations and as per patient demographic characteristics. Even though presently the evidence demonstrating acute pain relief and its long-term positive outcomes are lacking, every anaesthesiologist should prescribe optimal multimodal analgesia and add RA technique wherever feasible. The issues with currently analyzed data are small number studies, performance bias, attrition, and incomplete outcome data especially at 12 months postoperatively. This should not prohibit clinicians in adding RA technique as an important component of MMA to all surgical patients. 
1. Bowyer AJ, Royse CF. Postoperative recovery and outcomes--what are we measuring and for whom? Anaesthesia. 2016;71 Suppl 1:72-7.

2. Kettner SC, Willschke H, Marhofer P. Does regional anaesthesia really improve outcome? BrJAnaesth. 2011;107 Suppl 1: i90-5.

3. Small C, Laycock H. Acute postoperative pain management. BrJ Surg. 2020 ;107:e70-e80.

4. Gan TJ. Poorly controlled postoperative pain: prevalence, consequences, and prevention.JPain Res. 2017; 10:2287-98.

5. Kessler J, Marhofer P, Hopkins PM, Hollmann MW. Peripheral regional anaesthesia and outcome: lessons learned from the last 10 years. Br J Anaesth. $2015 ; 114: 728-45$.

6. Milosavljevic SB, Pavlovic AP, Trpkovic SV, Ilić AN, Sekulic AD. Influence of spinal and general anesthesia on the metabolic, hormonal, and hemodynamic response in elective surgical patients. Med Sci Monit. 2014; 20:1833-40.

7. Rawal N. Current issues in postoperative pain management. Eur J Anaesthesiol.2016; 33:160-71.

8. Moon YE. Postoperative nausea and vomiting. Korean J Anesthesiol. 2014; 67:164-170

9. Gan TJ, Belani KG, Bergese S, Chung F, Diemunsch P, Habib AS et al. Fourth Consensus Guidelines for the Management of Postoperative Nausea and Vomiting. Anesth Analg. 2020; 131:411-48.

10. Li J, Zhu Y, Chen W, Zhao K, Zhang J, Meng $H$ et al. Incidence and locations of deep venous thrombosis of the lower extremity following surgeries of tibial plateau fractures: a prospective cohort study. J Orthop Surg Res. 2020; 15:605.

11. Wang H, Kandemir U, Liu P, Zhang H, Wang PF, Zhang BF, Shang K, Fu $Y H$, Ke C, Zhuang $Y$, Wei X, Li Z, Zhang K. Perioperative incidence and locations of deep vein thrombosis following specific isolated lower extremity fractures. Injury. 2018; 49:1353-7.

12. Smith LM, Cozowicz C, Uda Y, Memtsoudis SG, Barrington MJ. Neuraxial and Combined Neuraxial/General Anesthesia Compared to General Anesthesia for Major Truncal and Lower Limb Surgery: A Systematic Review and Meta-analysis. Anesth Analg. 2017; 125:1931-45.

13. Johnson RL, Kopp SL, Burkle CM, Duncan CM, Jacob AK, Erwin PJ, Murad MH, Mantilla CB. Neuraxial vs general anaesthesia for total hip and total knee arthroplasty: a systematic review of comparative-effectiveness research.BrJAnaesth. 2016; 116:163-76.

14. Pozek JP, Beausang D, Baratta JL, Viscusi ER. The Acute to Chronic Pain Transition: Can Chronic Pain Be Prevented? Med Clin North Am. 2016; 100:17-30.

15. Ahuja V, Thapa D, Ghai B. Strategies for prevention of lower limb postamputation pain: A clinical narrative review. J Anaesthesiol Clin Pharmacol. 2018; 34:439-49.

16. Wang $W$, Wang $Y, W u H$, et al. Postoperative cognitive dysfunction: current developments in mechanism and prevention. Med Sci Monit. 2014; 20:190812.

17. Kotekar N, Kuruvilla CS, Murthy V. Post-operative cognitive dysfunction in the elderly: A prospective clinical study. Indian J Anaesth. 2014; 58:263-8.

18. Mason SE, Noel-Storr A, Ritchie CW. The impact of general and regional anesthesia on the incidence of post-operative cognitive dysfunction and postoperative delirium: a systematic review with meta-analysis. J Alzheimers Dis.
2010;22 Suppl3:67-79.

19. Davis N, Lee M, Lin AY, et al. Postoperative cognitive function following general versus regional anesthesia: a systematic review. J Neurosurg Anesthesiol.2014; 26:369-76.

20. Zywiel MG, Prabhu A, Perruccio AV, Gandhi R. The influence of anesthesia and pain management on cognitive dysfunction after joint arthroplasty: a systematic review. Clin Orthop Relat Res. 2014; 472:1453-66.

21. Nair AS, Naik V, Saifuddin MS, Narayanan H, Rayani BK. Regional anesthesia prevents cancer recurrence after oncosurgery! What is wrong with the hypothesis? Indian J Cancer 2020 ; XX: XX-XX. 10.4103/ijc.IJC_331_20(Accepted manuscript)

22. Divatia JV, Ambulkar R. Anesthesia and cancer recurrence: What is the evidence? J Anaesthesiol Clin Pharmacol 2014; 30:147-50.

23. Yap A, Lopez-Olivo MA, Dubowitz J, Hiller J, Riedel B. Global Onco-Anesthesia Research Collaboration Group. Anesthetic technique and cancer outcomes: A meta-analysis of total intravenous versus volatile anesthesia. CanJAnaesth 2019; 66:546-61.

24. Sessler DI, Pei L, Huang Y, Fleischmann E, Marhofer P, Kurz A et al; Breast Cancer Recurrence Collaboration. Recurrence of breast cancer after regional or general anaesthesia: a randomised controlled trial. Lancet. 2019;394(10211):1807-15.

25. Neal JM. Ultrasound-Guided Regional Anesthesia and Patient Safety: Update of an Evidence-Based Analysis. Reg Anesth Pain Med. 2016; 41:195204.

26. Topor B, Oldman M, Nicholls B. Best practices for safety and quality in peripheral regional anaesthesia. BJAEduc. 2020; 20:341-7.

27. Shivaprakash S, Diwan SM | Anatomy of Brachial Plexus Above The Clavicle |International Journal of Regional Anaesthesia | January-June 2021; 2(1):29-34.

28. Sivashanmugam, Areti A | Volume of Local Anaesthetic Agents and Block Efficacy in Blocks Above the Clavicle International Journal of Regional Anaesthesia|January-June 2021;2(1):35-38.

29. Mirle R, Mukundan S | Cadaveric Workshop and Implications in Regional Anaesthesia | International Journal of Regional Anaesthesia | January-June $2021 ; 2(1): 22-28$.

30. Gopal TVS, Maniar A, Chakraborty A, Kulkarni R | Abdominal WallBlocks in Abdominal Surgery: An Update | International Journal of Regional Anaesthesia |January-June 2021; 2(1): 47-53.

31. Chhabra A, Sethi D, Nair A | Regional Anaesthesia for Breast surgery |International Journal of Regional Anaesthesia | January-June 2021; (1): 4046.

32. Giri S | Landmark and PNS Guided Forearm Blocks | International Journal of Regional Anaesthesia|January-June 2021; 2(1):67-71.

33. Murlidhar TS, Vadera H IAORA4U-A Regional Anaesthesia App for AORA Members | International Journal of Regional Anaesthesia | JanuaryJune $2021 ; 2(1): 72-73$.

34. Kulkarni R, Maniyar A, Singh N, Gupta K, Ponde V| Sterility Protocols During Regional Anaesthesia |International Journal of Regional Anaesthesia | January-June 2021; 2(1):05-12.

35. Grewal A | Case Report: Pearls and Pitfalls | International Journal of Regional Anaesthesia |January-June 2021; 2(1): 19-21. 